\title{
An optimized genome-wide, virus-free CRISPR screen for mammalian cells
}

Xiong, Kai; Karottki, Karen Julie la Cour; Hefzi, Hooman; Li, Songyuan; Grav, Lise Marie; Li, Shangzhong; Spahn, Philipp; Lee, Jae Seong; Ventina, Ildze; Lee, Gyun Min

Total number of authors:

13

Published in:

Cell Reports Methods

Link to article, DOI:

10.1016/j.crmeth.2021.100062

Publication date:

2021

Document Version

Publisher's PDF, also known as Version of record

Link back to DTU Orbit

Citation (APA):

Xiong, K., Karottki, K. J. L. C., Hefzi, H., Li, S., Grav, L. M., Li, S., Spahn, P., Lee, J. S., Ventina, I., Lee, G. M., Lewis, N. E., Kildegaard, H. F., \& Pedersen, L. E. (2021). An optimized genome-wide, virus-free CRISPR screen for mammalian cells. Cell Reports Methods, 1(4), [100062]. https://doi.org/10.1016/j.crmeth.2021.100062

\section{General rights}

Copyright and moral rights for the publications made accessible in the public portal are retained by the authors and/or other copyright owners and it is a condition of accessing publications that users recognise and abide by the legal requirements associated with these rights.

- Users may download and print one copy of any publication from the public portal for the purpose of private study or research.

- You may not further distribute the material or use it for any profit-making activity or commercial gain

- You may freely distribute the URL identifying the publication in the public portal 


\section{Cell Reports \\ Methods}

\section{An optimized genome-wide, virus-free CRISPR screen for mammalian cells}

\section{Graphical abstract}

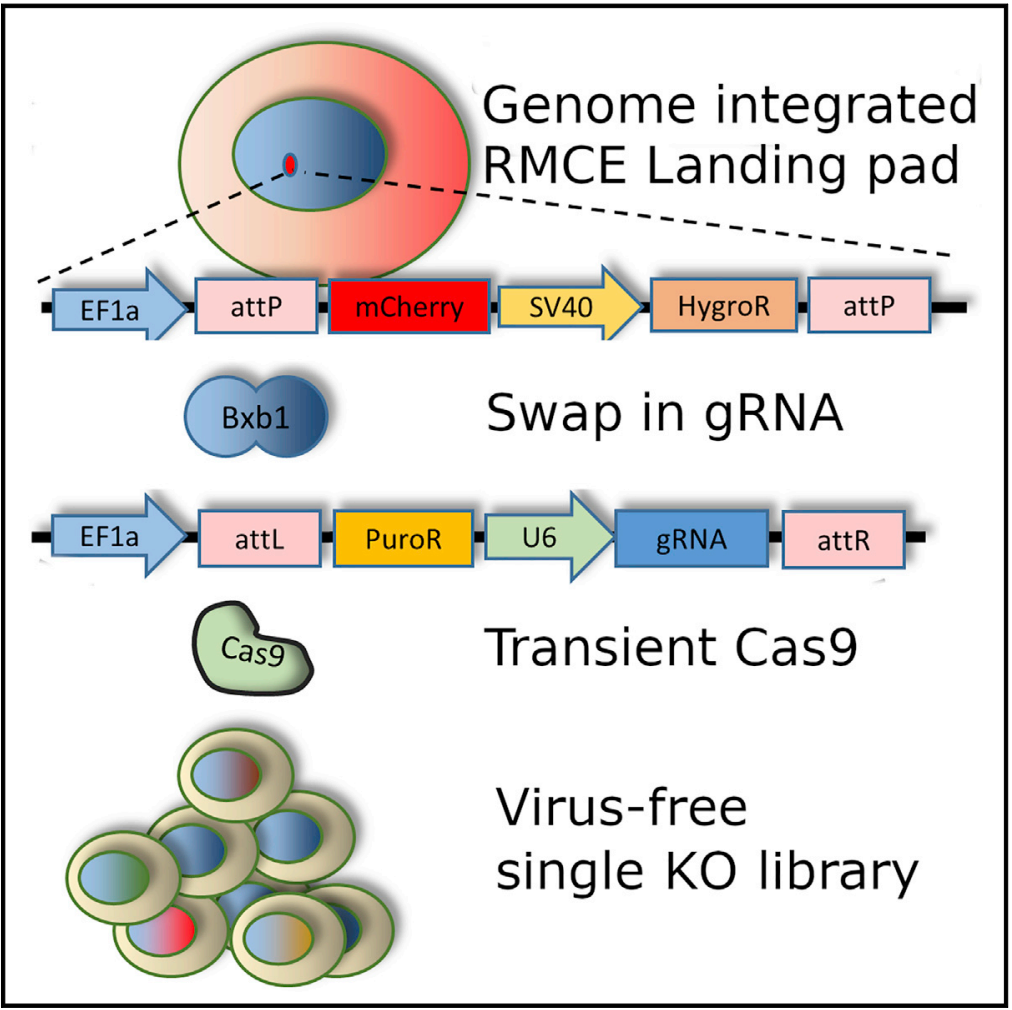

\section{Highlights}

- A recombinase-based method for virus-free, genome-wide CRISPR knockout screens

- Utilizes the same libraries and analysis tools as traditional virus-based screens

- Avoids noise associated with virus-based random insertions into the genome

\section{Authors}

Kai Xiong, Karen Julie la Cour Karottki, Hooman Hefzi, ..., Nathan E. Lewis, Helene Faustrup Kildegaard, Lasse Ebdrup Pedersen

\section{Correspondence}

laeb@dtu.dk

\section{In brief}

CRISPR knockout screening has mostly been performed by using viruses to deliver the required components into cells. In this paper, Xiong et al. demonstrate a virus-free approach that reduces noise and broadens access to CRISPR-based screens. 


\title{
Article \\ An optimized genome-wide, virus-free CRISPR screen for mammalian cells
}

\author{
Kai Xiong, ${ }^{1}$ Karen Julie la Cour Karottki, ${ }^{1}$ Hooman Hefzi, ${ }^{2,5}$ Songyuan Li, ${ }^{1}$ Lise Marie Grav, ${ }^{1}$ Shangzhong Li, ${ }^{2,6}$ \\ Philipp Spahn, ${ }^{2,5}$ Jae Seong Lee, ${ }^{3}$ Ildze Ventina, ${ }^{1}$ Gyun Min Lee, ${ }^{1,4}$ Nathan E. Lewis, ${ }^{2,5,6}$ Helene Faustrup Kildegaard, ${ }^{1}$ \\ and Lasse Ebdrup Pedersen $1,7,8, *$ \\ ${ }^{1}$ The Novo Nordisk Foundation Center for Biosustainability, Technical University of Denmark, Lyngby, Denmark \\ ${ }^{2}$ The Novo Nordisk Foundation Center for Biosustainability, University of California at San Diego, La Jolla, CA, USA \\ ${ }^{3}$ Department of Molecular Science and Technology, Ajou University, Suwon 16499, Republic of Korea \\ ${ }^{4}$ Department of Biological Sciences, KAIST, Daejeon, Republic of Korea \\ ${ }^{5}$ Department of Pediatrics, University of California at San Diego, La Jolla, CA, USA \\ ${ }^{6}$ Department of Bioengineering, University of California at San Diego, La Jolla, CA, USA \\ ${ }^{7}$ Department of Bioengineering, Technical University of Denmark, Lyngby, Denmark \\ 8Lead contact \\ *Correspondence: laeb@dtu.dk \\ https://doi.org/10.1016/j.crmeth.2021.100062
}

MOTIVATION Although lentivirus-based delivery of genome-wide CRISPR screen components has proven successful, there are situations in, e.g., industry and hospitals where working with live viruses is difficult or simply not an option. For those situations we have developed an alternative to virus-based, genome-wide CRISPR screens that retains compatibility with the software tools developed for analyzing the results, takes a similar amount of time, and offers improved signal-to-noise ratio.

\section{SUMMARY}

Pooled CRISPR screens have been widely applied to mammalian and other organisms to elucidate the interplay between genes and phenotypes of interest. The most popular method for delivering the CRISPR components into mammalian cells is lentivirus based. However, because lentivirus is not always an option, virus-free protocols are starting to emerge. Here, we demonstrate an improved virus-free, genome-wide CRISPR screening platform for Chinese hamster ovary cells with 75,488 gRNAs targeting 15,028 genes. Each gRNA expression cassette in the library is precisely integrated into a genomic landing pad, resulting in a very high percentage of single gRNA insertions and minimal clonal variation. Using this platform, we perform a negative selection screen on cell proliferation that identifies 1,980 genes that affect proliferation and a positive selection screen on the toxic endoplasmic reticulum stress inducer, tunicamycin, that identifies 77 gene knockouts that improve survivability.

\section{INTRODUCTION}

CRISPR screens have been widely applied to decipher mammalian gene function at the genome scale, but most rely on lentiviral delivery methods (Adamson et al., 2016; Joung et al., 2017; Liu et al., 2018). However, lentivirus is not always an option. Working with lentivirus requires specialized facilities as well as trained personnel, which are not available in all laboratories. In some industrial and medical facilities, it is likewise considered an increased risk to work with live viruses. As we demonstrate in this paper, a recombinase-mediated cassette exchange (RMCE)-based, virus-free (VF) method can not only eliminate those concerns, but also result in less noisy data. RMCE is a method in which one can move a piece of DNA, for example, from a plasmid, into a pre-established genome landing pad without causing large-scale disruptions that can occur with CRISPR-mediated targeted insertion.

VF CRISPR screens are starting to emerge using various alternative strategies to perform the screen and detect hits. Examples are replacing an integrated dummy guide RNA (gRNA) with a pooled library of gRNAs by using homologous recombination (Rajagopal et al., 2016) or performing whole-genome sequencing to detect mutations caused by transient CRISPR expression (Kim et al., 2017). However, these strategies suffer from drawbacks. The method based on homologous recombination reports that the efficiency of the gRNA cassette integration is much lower than lentiviral integration efficiency, and it cannot be enriched by selection because of a risk of random integration of promoter-less gRNA that complicates "hit detection." This makes it difficult to perform very large screens because of the 


\section{Cell Reports Methods}

very large number of cells needed (Rajagopal et al., 2016). The second method, applying whole-genome sequencing to detect CRISPR-caused mutations in a pool of cells, is in our opinion more promising, but reliably linking such mutations to phenotype is not trivial, especially when using genomically unstable cell lines such as HEK, HeLa, other cancer cell lines, or Chinese hamster ovary $(\mathrm{CHO})$ cells and, in addition, full genome sequencing will need to be performed at a very high depth to reliably detect any effect less than "extreme" (Kim et al., 2017).

In this study, we present a new VF CRISPR screening platform and demonstrate it in $\mathrm{CHO}$ cells, the model of choice for producing pharmaceutical proteins (Walsh, 2018). We apply this platform to investigate key phenotypes in protein production contexts, namely, cell proliferation and ER stress resistance.

We obtain an in-cell library that covers $99.95 \%$ of our 75,488 gRNA large library with $92.5 \%$ of the cells containing exactly one gRNA. We show that this VF approach has low noise and works for both depletion and enrichment studies.

\section{RESULTS}

Design of the virus-free, genome-wide CRISPR pooled screening platform

Using 1,558 RNA-sequencing (RNA-seq) samples from different $\mathrm{CHO}$ cell lines and culture conditions, we selected $\sim 15,000$ expressed genes from the $\mathrm{CHO}$ genome (Rupp et al., 2018). Then, we designed gRNAs against the coding sequences of these genes (Table S1). These gRNAs were used to build a genomewide VF library that contained 75,488 unique gRNAs, of which 72,149 target 15,028 genes, 2,218 target intergenic regions, 1,051 are non-targeting (NT) gRNAs, and finally 70 gRNAs target multiple genes (Figure 1A, Table S1).

In the VF screening platform, we used Bxb1 RMCE to precisely integrate a single gRNA expression cassette. We made a $\mathrm{CHO}-\mathrm{S}$ cell line with a Bxb1 RMCE landing pad that contains an EF1a promoter followed by a recombinase target site (attP), an mCherry expression unit, and an SV40 promoter driving expression of a hygromycin resistance gene (HygroR) followed by the second recombinase target site (mutant attP). The integration site of this landing pad was previously selected as an active site surrounded by highly expressed genes (Petersen et al., 2018; Pristovšek et al., 2019; Xiong et al., 2019). This cell line is termed CHO-attp-mCherry. In the donor gRNA library plasmid, the gRNA expression cassette for RMCE is flanked by recombinase target sites (attB and mutant attB). To allow for enrichment of the integrated gRNA while also preventing enrichment of randomly integrated gRNAs, we placed a promoter-less puromycin resistance gene (PuroR) within the recombination site so that the PuroR gene is expressed only after correct insertion into the landing pad with the EF1a promoter upstream. Lack of mCherry expression thus indicates a successful RMCE of the gRNA cassette. After transfection with the Bxb1 recombinase plasmid and gRNA library plasmids, $\sim 4 \%$ of the cells demonstrated successful RMCE (Figure S1). The RMCE-positive cells were then further enriched by puromycin $(10 \mu \mathrm{g} / \mathrm{mL})$ selection for 14-20 days (Figure 2). Twenty-five days after gRNA RMCE, the RMCE cell population was fully enriched, as evidenced by $99.78 \%$ of the cells being negative for mCherry (Figure 1C).
Spurious gene editing is reduced by using transiently expressed Cas9

Spurious gene editing can occur by transient expression of gRNAs from plasmids after transfection of the gRNA library if Cas9 is simultaneously expressed (Figure $3 \mathrm{~A}$ ). To overcome this issue, instead of using a stable Cas9-expressing cell line, we used transient Cas9 expression in the gRNA RMCE cell pool, introduced after cell-division-based dilution of non-integrated gRNA plasmids. To confirm that spurious gene editing was avoided using this strategy, we performed a test to compare the transient strategy with a cell line stably expressing Cas9. gRNA targeting Mgat1 (Mgat1gRNA) and an NT-gRNA were mixed at the ratio 1:1. This mixture of gRNAs was co-transfected with Bxb1 into $\mathrm{CHO}$-attp-mCherry cells. Ten days after transfection of the gRNA, the CHO-attpmCherry cells were transiently transfected with Cas9, and meanwhile, the mixture of gRNAs was co-transfected with Bxb1 into $\mathrm{CHO}$ cells stably expressing Cas9 (CHO-attp-mCherry/Cas9). Two days later, both cell line populations were single-cell sorted into 96-well plates (Figure 3B). We then tested the cells for Mgat1 gene editing and gRNA integration in the landing pad by Sanger sequencing. Spurious gene editing would be a case in which Mgat1 was edited but the NT-gRNA was integrated in the landing pad (Figure $3 \mathrm{C}$ ). The sequencing analysis confirmed that there is no spurious gene editing when using transient Cas9, whereas undesired spurious editing was found in the stable Cas 9 cells, presumably because of gRNA transcribed from unintegrated gRNA plasmids (Figure 3D). We thus successfully avoided such undesired and hard-to-detect spurious gene editing. Knowing this, in the following library establishment, we transfected Cas9 25 days after transfecting the gRNA RMCE system to allow sufficient time for dilution/degradation of unintegrated gRNA expression plasmids.

Precise integration of gRNA expression cassette results in lower clonal variance in cell population and achieves high library coverage

While constructing and testing the VF library, we simultaneously ran experiments by using a previously established lentiviral library (Figure 1B) to verify that our VF library gave similar results.

We hypothesized that the targeted integration of gRNA might lead to less clonal variation than the randomly integrated gRNA in the lentiviral library. To investigate the effect of gRNA integration methods on the phenotype of $\mathrm{CHO}$ cells, before Cas 9 delivery, we analyzed the variance of cell proliferation in the lentiviral library and VF library by measuring the colony confluence 14 days after single-cell sorting into 96-well plates (Figure 1D). The cell population resulting from lentiviral library transduction showed higher variation across cells than the VF library population. We hypothesize that this higher clone variance in the lentiviral library population is caused by random, and potentially multiple, genomic integrations of gRNA.

To verify the desired single-copy integration of gRNA cassettes, we used qPCR to determine the copy number of genome-integrated gRNA cassettes in the VF RMCE cells and compared with the lentiviral library. Using VF RMCE, $92.5 \%$ of the cells showed single integration of gRNA cassettes, whereas the number of integration events in the lentiviral library followed a Poisson distribution (Figure 1E). Our previous study of the 
A

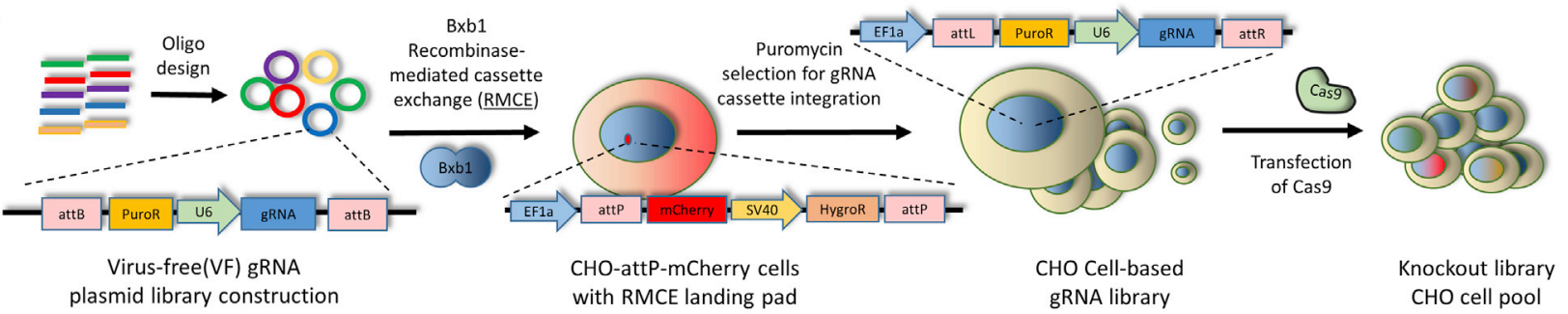

B

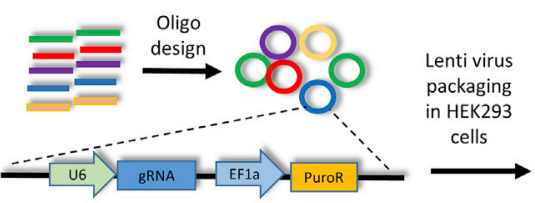

Lenti-gRNA plasmid

library construction

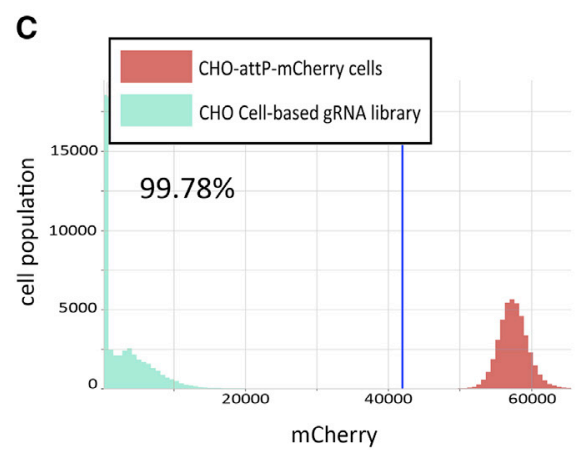

Lentivirus pool containing gRNA cassette
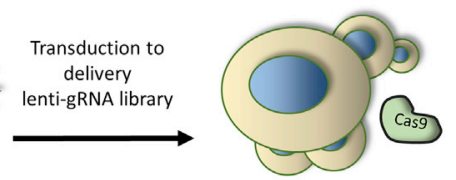

Puromycin selection for gRNA cassette integration

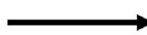

$\mathrm{CHO}$ cells with stable or transient Cas9 expression

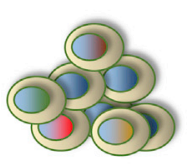

Knockout library $\mathrm{CHO}$ cell pool
D

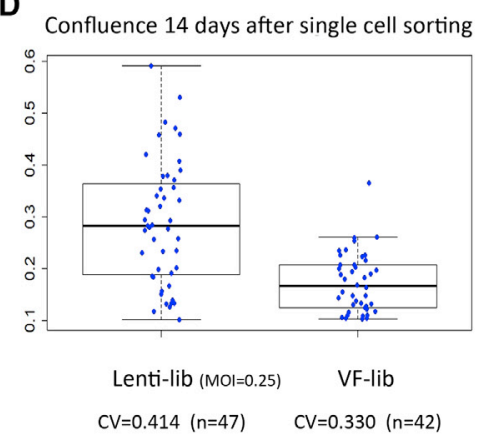

E

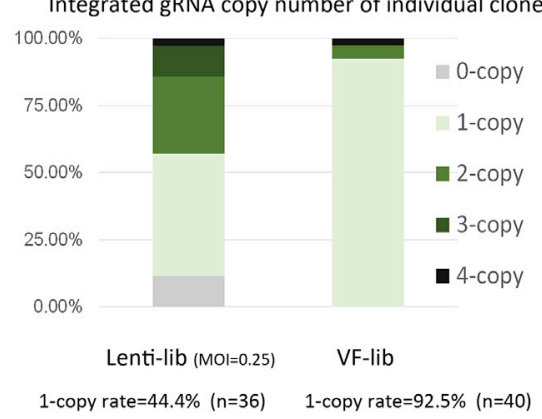

F

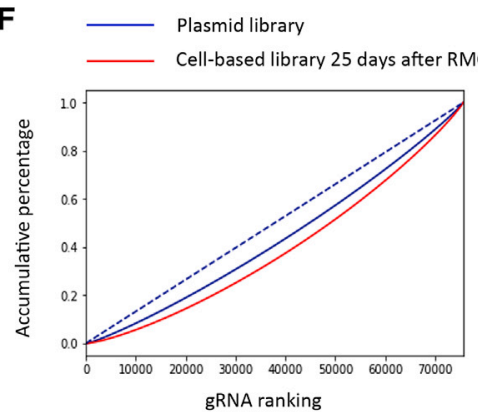

G

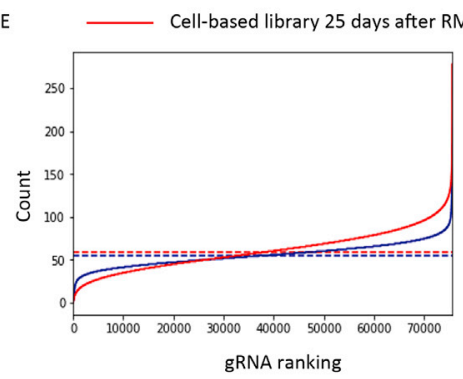

H

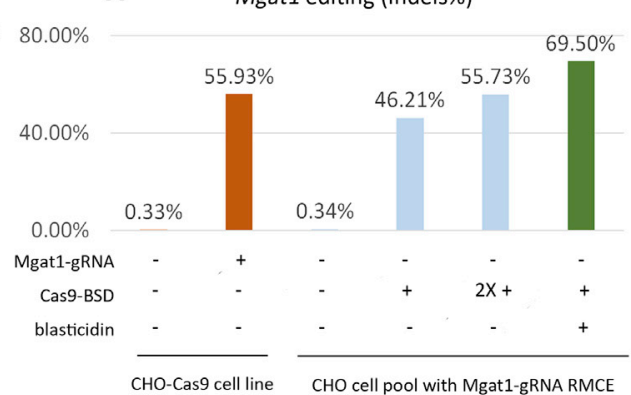

Figure 1. Establishing a virus-free (VF) CRISPR screen platform

(A and B) Shown are a (A) VF CRISPR screen and a (B) lentiviral CRISPR screen. A genome-wide VF library was designed, containing 74,617 gRNAs targeting 18,353 expressed genes in CHO-S cells with 1,000 non-targeting control gRNAs. The gRNA expression cassette was precisely integrated into the working cell line by Bxb1 RMCE. A promoter-less puromycin-resistance gene (PuroR) in the RMCE donor DNA enables the precise integration of a single gRNA expression cassette. The lentiviral library contained 15,645 gRNAs targeting 2,599 genes with 1,000 non-targeting control gRNAs (lentiviral library).

(C) In the VF library, the cell pool containing the gRNA library was enriched after 25 days of puromycin selection. The mCherry cassette was finally replaced by the gRNA expression cassette.

(D) Before Cas9 was delivered, the cell clone variance in the lentiviral library and VF library was compared by measuring the colony confluence 14 days after single-cell sorting into 96 -well plates. CV indicates coefficient of variation.

$(E$ and $F)$ The (E) gRNA copy number in the lentiviral library and VF library was further detected by qPCR. gRNA coverage of the VF library was calculated in (F). Shown are the cumulative percentage of reads in the plasmid library after vector construction (blue solid line) and in the cell-based library 25 days after RMCE (red solid line). Dashed line, ideal model in gRNA library design.

(G) The number of reads per gRNA in the plasmid library after vector construction (blue solid line) and in the cell-based library 25 days after RMCE (red solid line). Dashed blue line, ideal model in plasmid library (mean of reads). Dashed red line, ideal model in cell-based library 25 days after RMCE (mean of reads).

(H) Testing of Cas9 delivery method by using Mgat1 as the target gene. After a cell pool was established with RMCE by using gRNA targeting Mgat1 (Mgat1gRNA), the transient Cas9-BSD vector was transfected. The Cas9-transfected cells were enriched by treatment with blasticidin. The Mgat1 editing efficiency was verified by NGS. Transfection of one or two rounds $(2 \mathrm{X}+)$ of Cas9 in the $\mathrm{CHO}$ cell pool with RMCE of Mgat1 was set as control. 


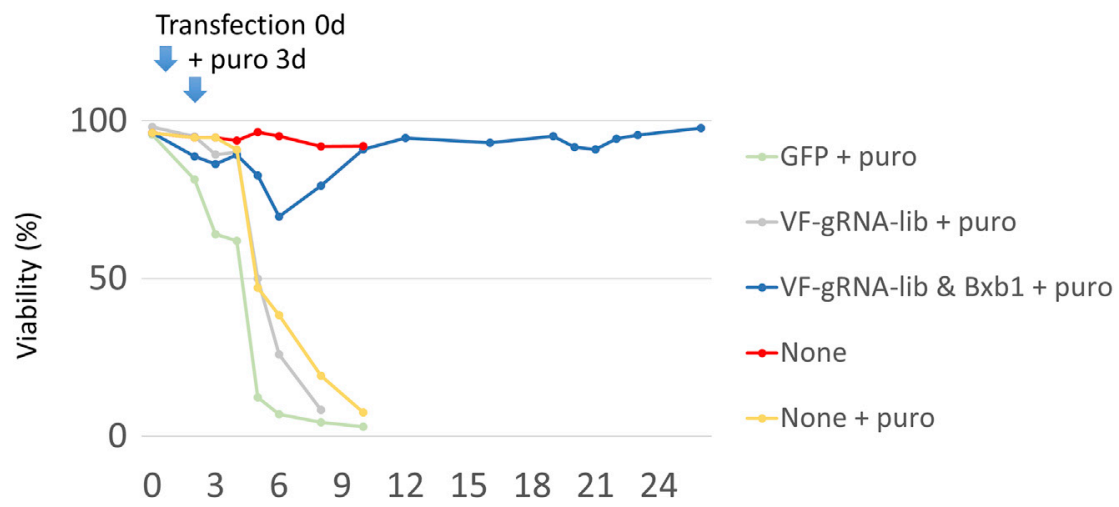

Figure 2. The cell viability during the process of establishing a VF cell-based gRNA library CHO-attp-mCherry cells with RMCE landing pads were co-transfected with Bxb1 recombinase and the VF gRNA library. Three days after transfection, cells were selected with $10 \mu \mathrm{g} / \mathrm{mL}$ puromycin for around 20 days. Cells transfected with GFP (GFP + puro) or without transfection (None + puro) were set as controls for puromycin selection. Cells transfected with only the VF gRNA library and without Bxb1 recombinase (VF-gRNA-lib + puro) confirm the limited random integration of gRNA cassettes. Nontreated cells (None) were used as a control.

Time after VF gRNA library RMCE (days)

splicing, and protein translation (Mi et al., 2019a, 2019b) (Figure 4B, Table S3). The significant depletion of gRNAs targeting 1,980

lentiviral library also demonstrated that it is common to have multiple different types of gRNAs in the cells that have multiple gRNA integrations. In summary, the VF integration results in low cloneto-clone variation in the cell pool before performing the screen.

The coverage of the gRNA library after integration was as high (99.95\%) in the cells as in the plasmid pool (99.98\%), and showed an even distribution of gRNAs (skew ratio [Konermann et al., 2015; Joung et al., 2017] = 1.85 and 2.84 in the plasmidand cell-based libraries, respectively, Figures $1 \mathrm{~F}$ and $1 \mathrm{G}$ ).

\section{Enriching for Cas9-transfected cells results in a high} gene editing rate

To identify an efficient method to deliver Cas9, we tested a vector expressing Cas 9 together with a blasticidin resistance gene (BSD) in $\mathrm{CHO}$ cells followed by treatment with blasticidin to kill the cells without transient Cas9 expression. Four days of blasticidin treatment was sufficient to kill untransfected cells (Figure S2). We verified this method via next-generation sequencing (NGS) by using the Mgat1 gene as a model. In the blasticidinselected Cas9-transfected CHO cells with RMCE of gRNA targeting Mgat1 (Mgat1-gRNA), Mgat1 gene editing efficiency was $\sim 70 \%$, which is higher than the efficiency via transfection of 1 or 2 rounds of Cas9-BSD without blasticidin selection. Also, the efficiency was higher than transient transfection of the gRNA into a $\mathrm{CHO}$ cell line with constitutive Cas9 expression (CHO-Cas9, Figure $1 \mathrm{H})$. With the protocol for enriching Cas9transfected cells now validated, we transfected the Cas9-BSD into the cells with the integrated gRNA library, and we subsequently followed with blasticidin selection to establish the final knockout (KO) CHO cell pool (Figures S2C and S2D).

\section{Genes essential for cell proliferation are identified in the VF CRISPR screen}

Nine and eighteen days after Cas 9 transfection, the genomic DNA from the cell pool was harvested for NGS analysis to measure the gRNA depletion. The percentage of depleted gRNAs increased after Cas9 transfection into the cell-based gRNA library (Figure 4A). Eighteen days after Cas9 transfection in the cell-based gRNA library, gene set enrichment analysis revealed significantly depleted genes in fundamental cellular processes: genes involved in DNA replication and repair, gene expression, RNA processing and genes was shown in three biologically independent samples compared with NT-gRNAs (Figure 4C and Table S4), indicating that these genes are likely essential to cell viability and/or proliferation under our culture conditions.

Using the essential gene database (www.essentialgene.org) (Luo et al., 2014), we compared our results to previous findings (Figures 4D and 4E, Table S5). Using all 20 human datasets from the essential gene database, we created a consensus set of essential genes consisting of all genes present in at least five of those studies. Compared with this consensus set, we found that 1,322 genes ( 47\%) are overlapping (Figure 4D). In addition, we analyzed previous pooled CRISPR KO screens in two human cell lines and found that K562 cells (Wang et al., 2017b) and HeLa cells (Hart et al., 2015) have similar amounts of overlap with each other as they do with our $\mathrm{CHO}$ data ( 37\% between $\mathrm{K} 562$ and $\mathrm{HeLa}, \sim 35 \%$ between $\mathrm{K} 562$ and $\mathrm{CHO}, \sim 33 \%$ between HeLa and $\mathrm{CHO}$ ) (Figure 4E, Table S5). From this we conclude that our VF CRISPR screen method can identify essential genes via gRNA depletion analysis as well as other libraries.

We also wanted to see how our results compared with a similar screen performed on our smaller lentivirus-based library. The overall fold change of targeted genes in both screens showed high correlation after Cas9 induction (Figure S3). The VF library and the lentiviral library have an overlap of 1,553 targeted genes, and of these, 344 were found to be significantly enriched in the lentiviral library and 286 were found in the VF library (Figure 4F). Of those genes, 228 (57\%) were identical, much higher than the overlap between the two human cell lines K562 and HeLa ( 37\%).

In summary, we identified 1,980 genes that have a negative impact on cell proliferation and/or viability. The list includes genes coding for key catalytic enzymes such as fumarate hydratase, NADH dehydrogenase, and fatty acid synthase (Tables S3 and S4). This information can be used to further understand cell metabolism and proliferation.

\section{Genes sensitive to induced ER stress are identified in the VF CRISPR screen}

Although many proteins, most notably antibodies, are readily produced in $\mathrm{CHO}$ cells, some proteins are currently difficult or impossible to produce. Such proteins are termed "difficult-toexpress" (DTE) or "difficult-to-produce" (DTP). Some of these 
A

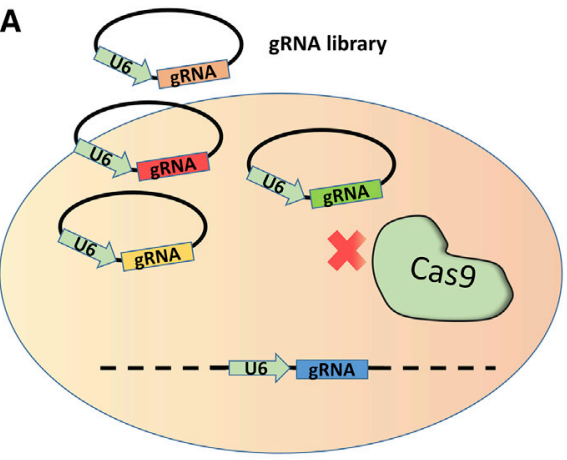

C

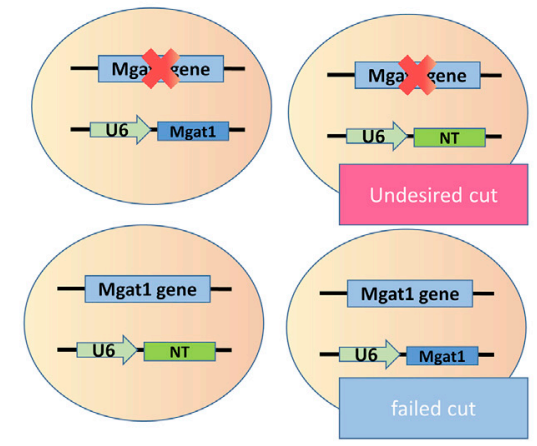

B

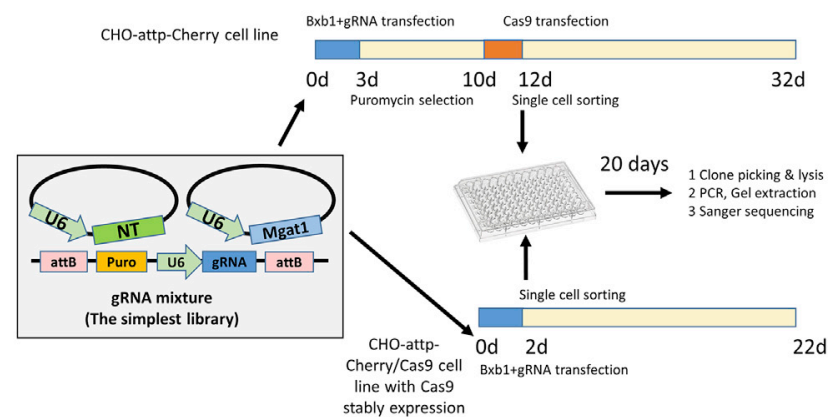

D

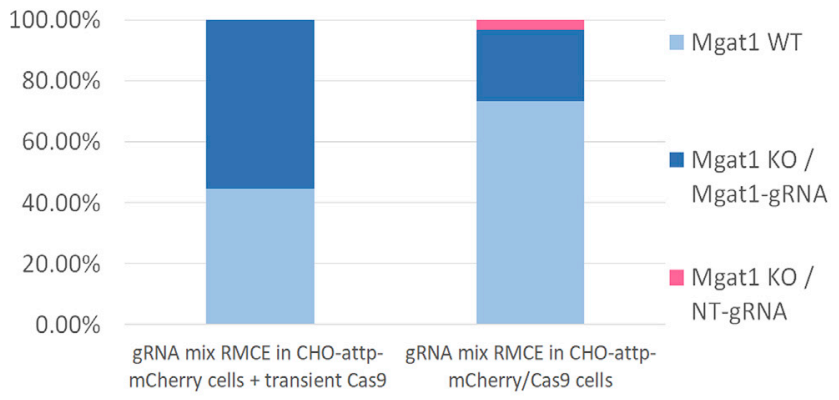

Figure 3. Detection of spurious gene editing in CRISPR

(A) Illustration of undesired spurious gene editing caused by the transfected gRNA library plasmid but not by the integrated gRNA cassette.

(B) Experimental design to compare the effects of spurious gene editing by transfected gRNA plasmid. The mixture of gRNAs containing Mgat1-gRNA and NTgRNA were co-transfected with Bxb1 into CHO-attP-mCherry cells, and 10 days afterward the Cas9 was transfected. The control was set as Bxb1 RMCE of the gRNA mixture in CHO-attP-mCherry/Cas9 cells in which Cas9 is stably expressed. Single cells from these two cell pools were sorted to form colonies for the following genomic DNA extraction.

(C) The gene editing and gRNA integration patterns were compared between the two protocols in (B).

(D) Percentage of different gene editing and gRNA integration patterns in two protocols: gRNA mix RMCE in CHO-attP-mCherry cells + transient Cas9 $(n=8)$ and gRNA mix RMCE in CHO-attP-mCherry/Cas9 cells $(n=30)$.

proteins result in high levels of unfolded proteins, which leads to the activation of the unfolded protein response (UPR) and ultimately leads to apoptosis (Prashad and Mehra, 2015). Some investigations indicate that reducing the UPR-mediated ER stress in $\mathrm{CHO}$ cells can improve protein productivity (Tigges and Fussenegger, 2006; Borth et al., 2008; Mohan and Lee, 2010; Le Fourn et al., 2014). As a simple demonstration of an enrichment screen, we used the ER-stress-inducing compound tunicamycin (TM) to simulate a DTE/DTP production situation. $\mathrm{CHO}$ cells grown in TM stop proliferating and ultimately die. By applying the VF CRISPR screen platform, we identified genes that mitigate the effect of $\mathrm{TM}$ on $\mathrm{CHO}$ cells.

The CRISPR KO CHO cell pool ( $60 \mathrm{~mL}$, starting density $7 \times 10^{5}$ cells $/ \mathrm{mL}$ ) was treated with TM $(20 \mathrm{ng} / \mathrm{mL})$ (Figure $5 \mathrm{~A})$. After 4 days of TM treatment, cells recovered for 5 days by being cultured in the medium without selection chemicals (Figure S4). The fold change in gRNAs in TM-treated and control cells was evaluated by NGS and computational analysis (Spahn et al., 2017), and the different fold-change patterns were observed in TM-treated and control groups (Figure 5B). We hypothesized that Mfsd2a (major facilitator domain containing 2A) would be a positive control, given that MFSD2A has been identified as an essential plasma membrane transporter of TM in a human cell line (Reiling et al., 2011) (Figure 5C). As expected, the gRNAs targeting Mfsd2a were significantly enriched after TM treatment (Figure 5D). In total, gRNAs targeting 77 genes enriched in this screen (Figure 5D, combined $p<0.01$ in Table S6). Network topology-based analysis (Liao et al., 2019) demonstrated that 36 of these candidate genes showed a high degree of protein-protein interactions (PPIs) between candidate genes (Figure 5E). Further analysis of PPIs by using the STRING database (Szklarczyk et al., 2019) demonstrated that these candidates were significantly clustered $\left(p=7.42 \times 10^{-8}\right.$; Figure S5).

We compared our VF screen results with those obtained by using the smaller lentiviral library. Here the overlap was less impressive than for the proliferation screen. When looking only at genes targeted by both libraries, 11 genes were found to be significant in the lentiviral library and 11 in the VF library, but only 1 overlapping gene, Degs 1 , was identified.

\section{Validation of candidate genes}

Degs1 (Delta 4-Desaturase, Sphingolipid 1) catalyzes the final step in the de novo biosynthesis of ceramides, and $\mathrm{KO}$ has been previously demonstrated to have an antiapoptotic effect 
A

Cell library + Cas9 Od

Cell library + Cas9 9d

- Cell library + Cas9 18d
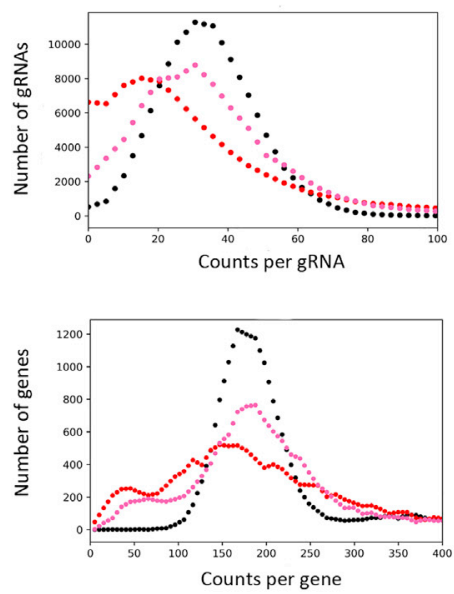

D

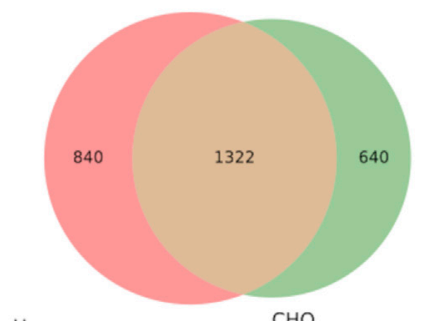

Human consensus

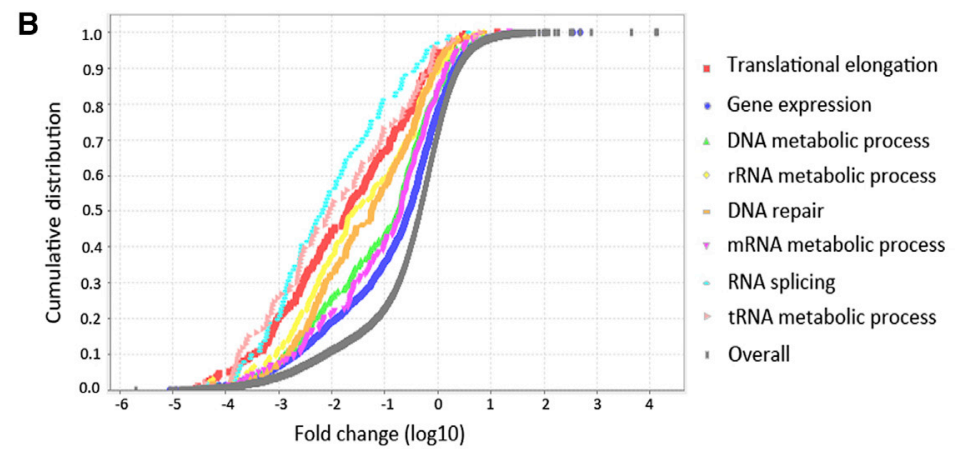

C

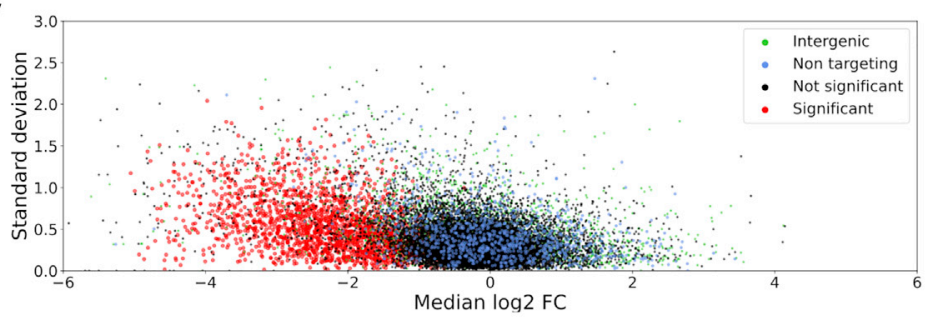

E

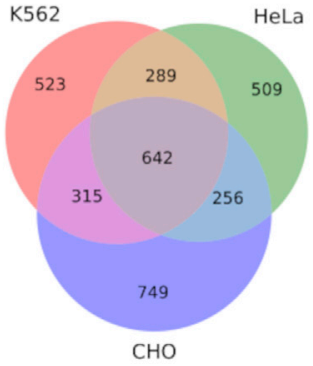

F

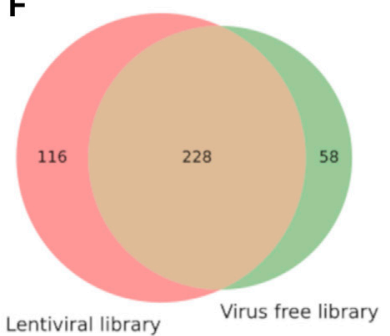

Figure 4. Genome-wide VF CRISPR screen to identify essential genes in CHO cells (A) gRNA read distribution of targeted genes after Cas9 transfection in the cell-based gRNA library.

(B) Selected biological processes significantly depleted in $\mathrm{CHO}$ cells 18 days after Cas 9 transfection in cell-based gRNA library ( $\mathrm{p}<0.05$ ).

(C-E) Shown are the (C) fold change (median of $\log _{2}$ ) and the standard deviation of gRNAs among three biological screening replicates. The significantly depleted gRNAs were classified as gRNAs targeting essential genes (highlighted in red). Venn diagrams of candidate essential genes in CHO cells and the reported essential genes (D) in human and (E) in K562 and HeLa cells.

(F) Overlap of essential genes between VF growth screen and lentiviral growth screen.

(Breen et al., 2013; Siddique et al., 2013; Rodriguez-Cuenca et al., 2015; Zhu et al., 2016). In the screens we performed, gRNAs targeting Degs1 were enriched after ER stress in both the VF screen and the lentivirus screen (Figure 6A). To confirm the effects caused by Degs $1 \mathrm{KO}$, we designed two additional gRNAs, also targeting Degs1, in addition to the five original gRNAs in the VF library (Figure 6B). Cas9-CHO cells with stable Cas9 expression were transfected with these two gRNAs, independently. Seven days after transfection, we treated the cell pools with TM $(20 \mathrm{ng} / \mathrm{mL})$ for an additional 7 days, and the cell density in each group was measured (Figure 6C). The cell growth curves indicated that the Cas9-CHO cells transfected with either gRNA targeting Degs1 survived better than the cells in the control groups. We further established two cell lines with isogenic Degs 1 KO by co-transfection of Cas 9 and gRNA-Degs 1 in wild-type $\mathrm{CHO}-\mathrm{S}$ cells followed by single-cell sorting and PCR detection and confirmed by Sanger sequencing (Figure 6D). Two clones were banked with +1 bp insertion near the gRNA tar- geting sequence (clone 1 was generated by gRNA1 and clone 2 was generated by gRNA2), and both of these Degs $1 \mathrm{KO} \mathrm{CHO}$ cell lines demonstrated resistance to TM-induced apoptosis compared with wild-type $\mathrm{CHO}-\mathrm{S}$ cells (Figure 6E).

In addition, we selected Bag6 (BCL2-Associated Athanogene) and Zfx (Zinc-Finger Protein X-Linked) for further validation, given that all the gRNAs targeting them significantly increased after TM treatment (Figure 5D). Two new gRNAs were designed targeting Bag6 and $Z f x$, respectively (Figure $7 A$ ). Seven days after transfection of the new gRNA for each gene into CHO-Cas9 cells with stable Cas 9 expression, the different cell pools were treated with TM $(20 \mathrm{ng} / \mathrm{mL})$ for an additional 4 days. Viability and viable cell density (VCD) improved for the CHO-Cas9 cells transfected with gRNAs targeting Bag6 or Zfx compared with the control cells, which were transfected with NT-gRNA or GFP. For example, the Bag 6 mutant recovered $88 \%$ of viability by day 4 after TM treatment (Figure 7B), and the VCD doubled compared with the control groups (Figure $7 \mathrm{C}$ ). Surprisingly, 
A

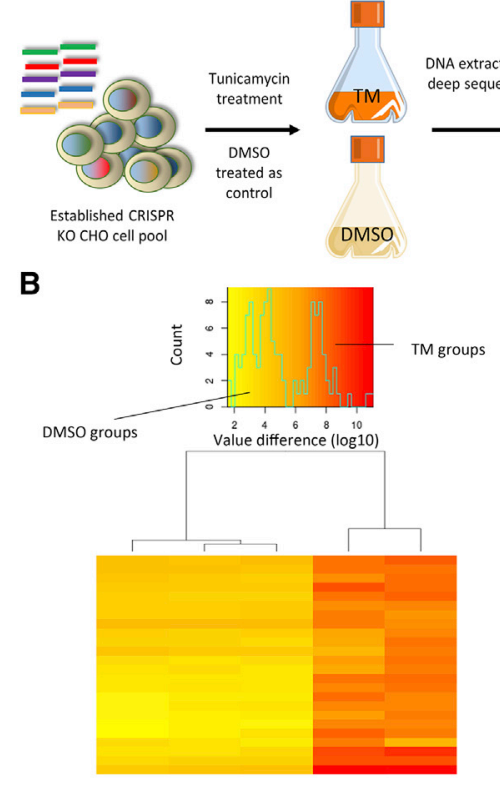

DMSO1 DMSO2 DMSO3 TM1 TM2
C
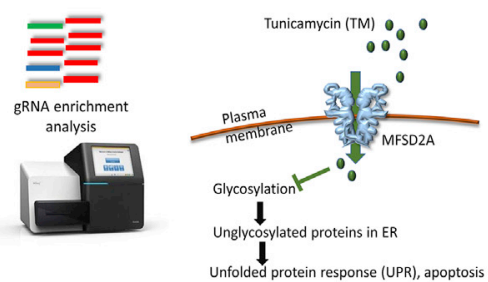

D

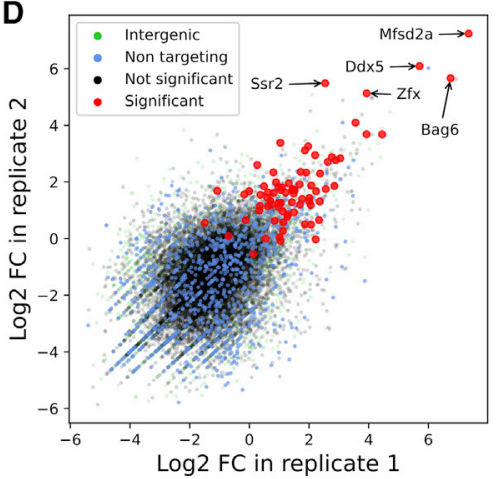

E

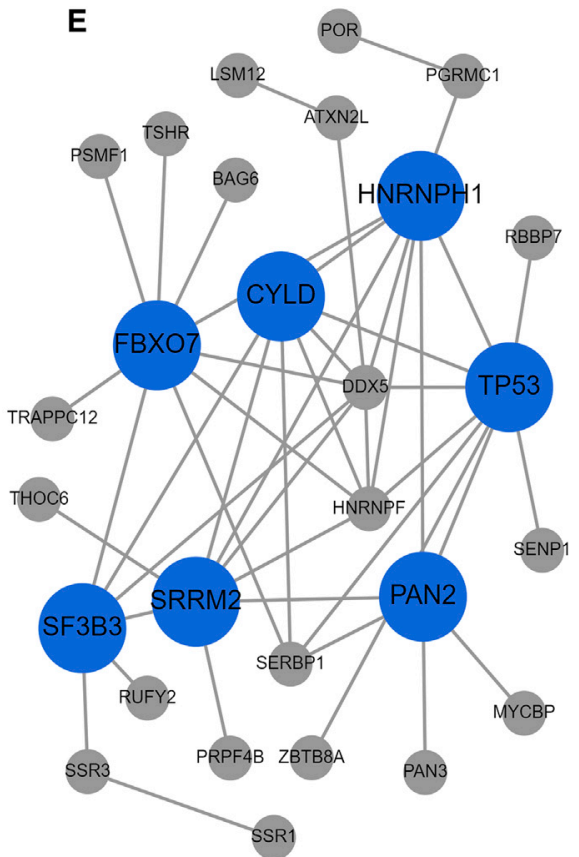

Figure 5. VF CRISPR screen for tunicamycin (TM)-resistant genes in $\mathrm{CHO}$ cells

(A) Illustration of screen. Apoptosis in $\mathrm{CHO}$ cells was induced by TM treatment $(20 \mathrm{ng} / \mathrm{mL})$, and cells treated with DMSO $(0.2 \%)$ were used as controls. (B) Heatmap of gRNA enrichment difference in TM-treated duplicates and control triplicates. This cluster analysis is based on the most abundant/depleted gRNAs. Log10 normalized read counts are color coded from lowest (yellow) to highest (red).

(C) Major facilitator domain containing 2A (MFSD2A) transporter as a key mediator in the response to TM.

(D) Fold change in gRNAs in VF CRISPR screen after TM treatment. Fold changes in the gRNAs targeting Bag6, and Zfx and the gRNAs targeting the positive control Mfsd2a are highlighted in red.

(E) Network-topology-based analysis based on the protein-protein interactions of candidate genes whose KO would provide TM resistance in CHO cells.

almost full resistance to TM was obtained in $\mathrm{CHO}$ cells after disruption of $Z f x$, wherein the cell viability after $Z f x$ disruption remained above $90 \%$ (Figure 7D) and the VCD was almost 5 times more than that of the control groups 4 days after TM treatment (Figure 7E). Furthermore, the expression levels of the $\mathrm{CHOP}$ gene, a well-known ER stress marker, significantly increased after TM treatment in control cells but not in the cells with $Z f x$ disruption (Figure 7F), indicating blockade of the ER stress pathway after Zfx KO. However, growth in TM-free culture medium showed that none these KOs gave an advantage to cell viability or VCD under normal conditions (Figure S6). We further investigated the effects of $Z f x \mathrm{KO}$ in isogenic $\mathrm{CHO}$ cell lines. We established three $Z f x$ KO CHO-S cell lines by single-cell sorting and confirming the $Z f x \mathrm{KO}$ with Sanger sequencing. Resistance to TM was likewise observed in these three Zfx KO cell lines (Figure $7 \mathrm{G}$ ). TM resistance obtained from $Z f x \mathrm{KO}$ was also observed in two established Enbrel-producing $\mathrm{CHO}$ cell lines (Sergeeva et al., 2020) (dual-RMCE ETN2_T9) with Zfx KO (Figure 7H). Thus, the VF CRISPR screen can successfully identify candidate genes via gRNA enrichment analysis.

\section{DISCUSSION}

Here we present a new and improved VF CRISPR screen approach with low noise that works with standard CRISPR screen analysis tools. In our VF system, $92.5 \%$ of cells demonstrated single integration of the gRNA expression cassette, thus decreasing the biases in screens caused by no or multiple integrations of gRNA (Figure 1E). Targeted integration also decreases clone-to-clone variance on productivity compared with random insertion (Lee et al., 2015). Similarly, we see in our VF platform significantly reduced clonal variation in cell proliferation compared with the random insertions in lentiviral methods (Figure 1D). The gRNA coverage in our cell-based genome-wide gRNA library was 99.95\% (Figure 1G). This is comparable to that achieved by a lentiviral strategy. Almost no gRNA expression cassettes were lost during the long-term cell culture (Figures $1 \mathrm{~F}$ and $1 \mathrm{G}$ ), indicating the stability of our platform. Therefore, our VF strategy improves on the genetic instability seen in lentiviral-transduced cells (Schambach et al., 2013; Rothe et al., 2014). In addition, the time cost for establishing the VF CRISPR screen platform is similar to that for establishing a lentiviral CRISPR screen platform (Figure S7).

Our platform also solved the problem that gRNA donor-integrated cells in the pool cannot be enriched by resistance-gene selection when random integration occurs. By applying Bxb1 RMCE to precisely integrate a donor-DNA-containing gRNA library with the promoter-less selection marker, the integrated cells could be enriched by puromycin selection in the final population, in which $99.78 \%$ of cells demonstrated successful integration of the gRNA expression cassette (Figure 1C). This 

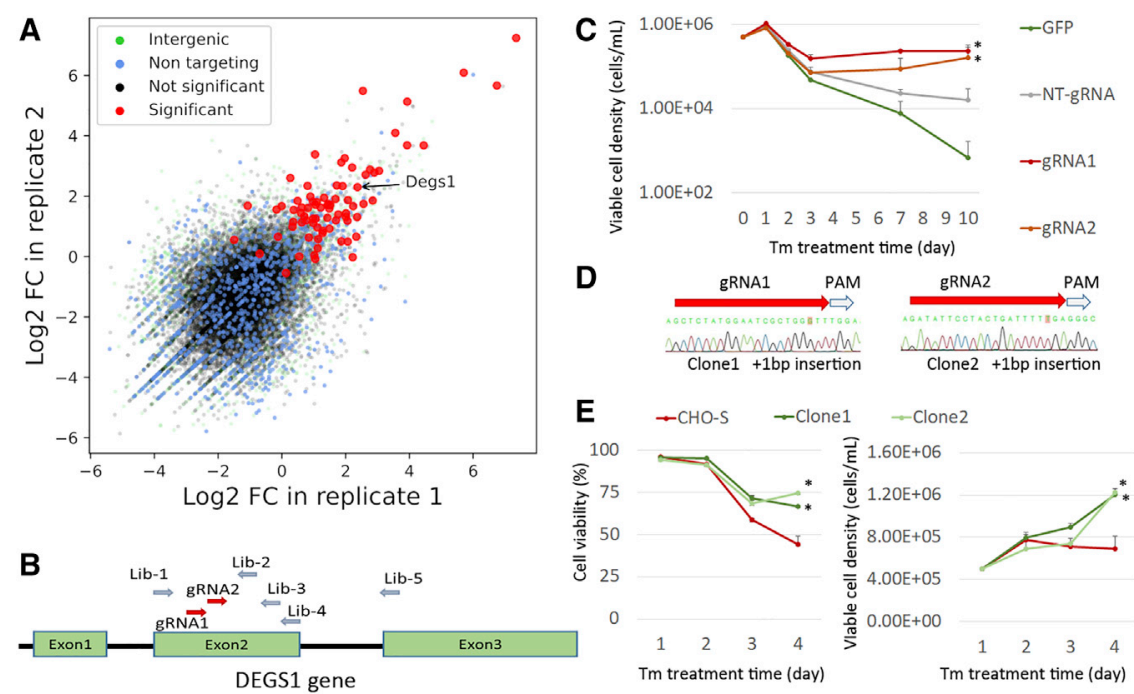

Figure 6. Validation of Degs1 as candidate gene for KO to obtain ER-stress resistance (A) Fold change in gRNAs targeting Degs 1 in two replicates of VF CRISPR screen after TM treatment.

(B) Two gRNAs were designed to target exon 2 of $D E G S 1$ in addition to the five gRNAs designed in the VF gRNA library.

(C) Effects of transfection of two gRNAs in Cas9$\mathrm{CHO}$ cells (continuously expressing Cas9) to obtain TM resistance. ${ }^{*} \mathrm{p}<0.05$. Statistical significance at day 10 .

(D and E) (D) Establishment of two cell lines with $D E G S 1 \mathrm{KO}$ and $(\mathrm{E})$ their resistance to TM treatment. ${ }^{*} p<0.05$. Statistical significance at day 4 .

former study showed that Bag6-null mouse cells are resistant to cell death induced by thapsigargin, which is another chemical that induces ER stress

efficiency is much higher than that achieved previously by a VF CRISPR screen approach based on homologous recombination, which showed an efficiency of around 30\% (Rajagopal et al., 2016).

For the application of the VF CRISPR screen in our study, Bag6 and Zfx are suggested for KO to reduce TM-induced apoptosis. Bag6 has previously been demonstrated to mediate apoptosis induced by ER stress (Desmots et al., 2008) and DNA damage (Sasaki et al., 2007; Krenciute et al., 2013). Similarly, a
(Desmots et al., 2008). Our study finds for the first time that Zfx $\mathrm{KO}$ provides absolute resistance to TM-induced apoptosis in $\mathrm{CHO}$ cells (Figure 7). It has previously been reported that TM treatment reduces the $\mathrm{N}$-glycosylation of $\mathrm{Zfx}$ and that the non-glycosylated form of Zfx accumulates in the cell, possibly being the cause of ER stress and/or apoptosis (Zhu et al., 2013). That hypothesis would explain why Zfx KO provides resistance to TM treatment. However, several previous studies showed that downregulating the Zfx gene generally results
A

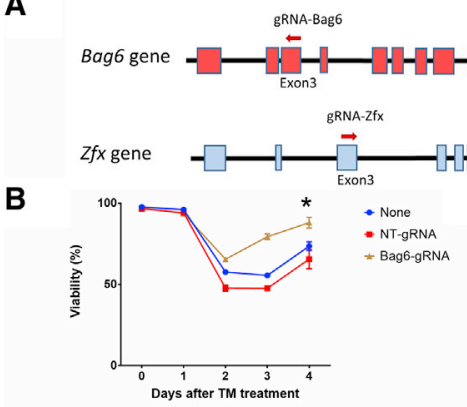

C

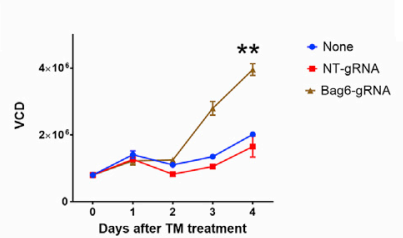

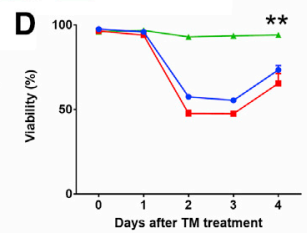

- None

$=$ NT-gRNA
$=$ Zfx-gRNA

E

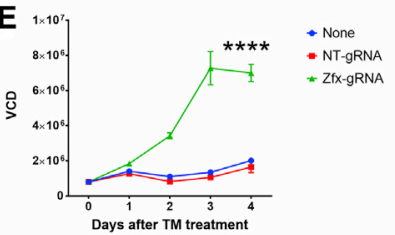

F
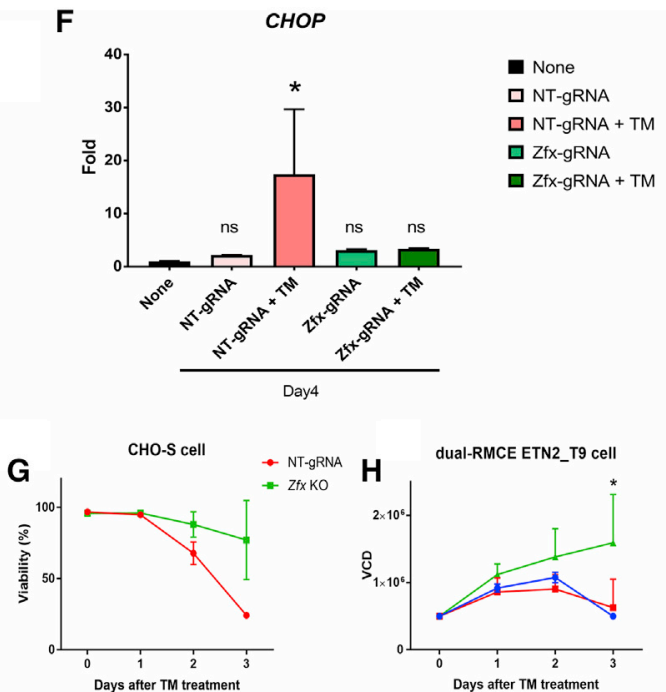

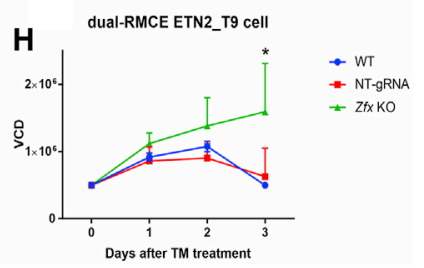

Figure 7. Validation of candidate genes for $\mathrm{KO}$ to obtain TM resistance

(A-E) Shown in (A), one new gRNA for each gene was designed to target Bag6 and Zfx. Effects of gRNA transfection in Cas9-CHO cells (constitutively expressing Cas9) on (B and D) cell viability and ( $C$ and $E$ ) viable cell density (VCD) targeting (B and C) Bag6 and (D and E) Zfx. Seven days after transfection of gRNA, the transfected Cas9-CHO cells were treated with $20 \mathrm{ng} / \mathrm{mL} \mathrm{TM}$, and the viability and VCD were recorded for 4 days afterward. Statistical analysis of data at day 4 was performed $\left({ }^{\star} \mathrm{p}<0.05,{ }^{* *} \mathrm{p}<0.01,{ }^{* \star *} \mathrm{p}<0.0001\right.$ compared with "None" group).

$(\mathrm{F}-\mathrm{H})$ As shown in $(\mathrm{F})$, the ER stress marker CHOP expression was measured at day 4 after TM treatment in (D) and (E), or at day 4 after cell seeding in Figure S8. Effects of $Z f x K O$ to obtain TM resistance in isogenic cell lines with Zfx KO using the parent cell line from $(G) C H O-S$ cells $(n=3)$ or from $(H)$ an Enbrel-producing cell line (dual-RMCE ETN2_T9, $n=2,{ }^{*} p<0.05$ compared with wild type ["WT"] group). The control cell lines were established by transfection with NT-gRNA and otherwise processed identical to the $Z f x$ KO cell lines $(n=2$ in $[G]$ and $[H])$. 
in increased apoptosis in human A549 cells (Zha et al. 2013), malignant glioma cells (Zhou et al., 2011), B lymphocytes (Arenzana et al., 2009), and mouse embryonic stem cells and hematopoietic stem cells (Cellot and Sauvageau, 2007; Galan-Caridad et al., 2007), suggesting an incomplete understanding of the role of Zfx and the toxicity of unglycosylated Zfx.

In summary, this work establishes a genome-wide VF CRISPR screening strategy that we believe will be of interest in cases in which working with viruses for whatever reason is unfeasible. In addition, for $\mathrm{CHO}$ cells, we present an expressed genomewide library that can be used for depletion or enrichment screening of phenotypes of interest in pharmaceutical protein production. Although applied here to $\mathrm{CHO}$ cells as an example, this approach can also be applied to other cell-based models. Thus, this approach presents a valuable method for precise VF genome-wide screens.

\section{Limitations of the study}

The presented method does have some limitations. For one, it requires the host cell to be pre-engineered with a landing pad for the recombinase system to work. This makes it more difficult to work with primary cells and likewise cells that are difficult to transfect. One can also speculate that picking one specific genomic integration spot for the gRNA might specifically perturb phenotypes that are somehow affected by that particular genome location.

\section{STAR $\star M E T H O D S$}

Detailed methods are provided in the online version of this paper and include the following:

- KEY RESOURCES TABLE

- RESOURCE AVAILABILITY

$\bigcirc$ Lead contact

○ Materials availability

O Data and code availability

- EXPERIMENTAL MODEL AND SUBJECT DETAILS

$\bigcirc$ Cell culture and transfection

- METHOD DETAILS

$\bigcirc$ Expressed genes filtering and VF gRNA library design

- Lentiviral library generation

O CHO-attp-mCherry cell line generation with RMCE landing pad and the RMCE of gRNA expression cassette

Copy number analysis using qPCR

O Cas9-transfected cells enrichment

o Preparation for NGS

O Clone variance analysis

- Gene disruption validation

O Generation of $\mathrm{CHO}-$ Cas9 cell line and $\mathrm{CHO}-\mathrm{attP}$ mCherry/Cas9 cell line

gRNA design for Mgat1 $\mathrm{KO}$ and Mgat1 editing analysis

Establishment of Zfx KO cell lines

O Plasmid maps and sequences

- QUANTIFICATION AND STATISTICAL ANALYSIS

O NGS data analysis

\section{SUPPLEMENTAL INFORMATION}

Supplemental information can be found online at https://doi.org/10.1016/j. crmeth.2021.100062.

\section{ACKNOWLEDGMENTS}

We thank Nachon Charanyanonda Petersen and Saranya Nallapareddy for helping with the FACS and molecular cloning. This work was supported by generous funding from the Novo Nordisk Foundation provided to the Center for Biosustainability at the Technical University of Denmark (NNF10CC1016517 and NNF160C0021638) and from National Insitute of General Medical Sciences (NIGMS) (R35 GM119850 to N.E.L.).

\section{AUTHOR CONTRIBUTIONS}

Conceptualization, K.X. and L.E.P.; methodology, K.X., L.E.P., J.S.L., and P.S.; validation, I.V.; investigation, K.X.; resources, K.X., K.J.I.C.K., H.H., S.L., L.M.G., S.L., and P.S.; data curation, L.E.P.; writing - original draft, K.X.; writing - review \& editing, L.E.P. and N.E.L.; visualization, K.X. and L.E.P.; supervision, L.E.P. and N.E.L.; project administration, L.E.P. and G.M.L.; funding acquisition, N.E.L.

\section{DECLARATION OF INTERESTS}

The authors declare no competing interests.

Received: April 19, 2021

Revised: May 17, 2021

Accepted: July 9, 2021

Published: August 4, 2021; corrected online October 25, 2021

\section{REFERENCES}

Adamson, B., Norman, T.M., Jost, M., Cho, M.Y., Nuñez, J.K., Chen, Y., Villalta, J.E., Gilbert, L.A., Horlbeck, M.A., Hein, M.Y., et al. (2016). A multiplexed single-cell CRISPR screening platform enables systematic dissection of the unfolded protein response. Cell 167, 1867-1882.e21. https://doi.org/10. 1016/j.cell.2016.11.048.

Arenzana, T.L., Smith-Raska, M.R., and Reizis, B. (2009). Transcription factor Zfx controls BCR-induced proliferation and survival of B lymphocytes. Blood 113, 5857-5867. https://doi.org/10.1182/blood-2008-11-188888.

Borth, N., Mattanovich, D., Kunert, R., and Katinger, H. (2008). Effect of increased expression of protein disulfide isomerase and heavy chain binding protein on antibody secretion in a recombinant $\mathrm{CHO}$ cell line. Biotechnol. Prog. 21, 106-111. https://doi.org/10.1021/bp0498241.

Breen, P., Joseph, N., Thompson, K., Kraveka, J.M., Gudz, T.I., Li, L., Rahmaniyan, M., Bielawski, J., Pierce, J.S., Van Buren, E., et al. (2013). Dihydroceramide desaturase knockdown impacts sphingolipids and apoptosis after photodamage in human head and neck squamous carcinoma cells. Anticancer Res. 33, 77-84.

Cellot, S., and Sauvageau, G. (2007). Zfx: at the crossroads of survival and selfrenewal. Cell 129, 239-241. https://doi.org/10.1016/j.cell.2007.04.002.

Desmots, F., Russell, H.R., Michel, D., and McKinnon, P.J. (2008). Scythe regulates apoptosis-inducing factor stability during endoplasmic reticulum stress-induced apoptosis. J. Biol. Chem. 283, 3264-3271. https://doi.org/ 10.1074/jbc.M706419200.

Galan-Caridad, J.M., Harel, S., Arenzana, T.L., Hou, Z.E., Doetsch, F.K., Mirny, L.A., and Reizis, B. (2007). Zfx controls the self-renewal of embryonic and hematopoietic stem cells. Cell 129, 345-357. https://doi.org/10.1016/j.cell.2007. 03.014.

Hart, T., Chandrashekhar, M., Aregger, M., Steinhart, Z., Brown, K.R., MacLeod, G., Mis, M., Zimmermann, M., Fradet-Turcotte, A., Sun, S., et al. (2015). High-resolution CRISPR screens reveal fitness genes and 
$\infty$ CellPress

OPEN ACCESS

Cell Reports Methods Article

genotype-specific cancer liabilities. Cell 163, 1515-1526. https://doi.org/10. 1016/j.cell.2015.11.015.

Joung, J., Konermann, S., Gootenberg, J.S., Abudayyeh, O.O., Platt, R.J., Brigham, M.D., Sanjana, N.E., and Zhang, F. (2017). Genome-scale CRISPR-Cas9 knockout and transcriptional activation screening. Nat. Protoc. 12, 828-863. https://doi.org/10.1038/nprot.2017.016.

Karottki, K.J.la C., Hefzi, H., Li, S., Pedersen, L.E., Spahn, P.N., Joshi, C., Ruckerbauer, D., Bort, J.A.H., Thomas, A., Lee, J.S., et al. (2021). A metabolic CRISPR-Cas9 screen in Chinese hamster ovary cells identifies glutamine-sensitive genes. Metab. Eng. 66,114-122. https://doi.org/10.1016/j.ymben.2021. 03.017.

Karottki, K.J.la C., Hefzi, H., Xiong, K., Shamie, I., Hansen, A.H., Li, S., Pedersen, L.E., Li, S., Lee, J.S., Lee, G.M., et al. (2020). Awakening dormant glycosyltransferases in $\mathrm{CHO}$ cells with CRISPRa. Biotechnol. Bioeng. 117, 593-598. https://doi.org/10.1002/bit.27199.

Kim, H.S., Lee, K., Bae, S., Park, J., Lee, C.-K., Kim, M., Kim, E., Kim, M., Kim, S., Kim, C., et al. (2017). CRISPR/Cas9-mediated gene knockout screens and target identification via whole-genome sequencing uncover host genes required for picornavirus infection. J. Biol. Chem. 292, 10664-10671. https:// doi.org/10.1074/jbc.M117.782425.

Konermann, S., Brigham, M.D., Trevino, A.E., Joung, J., Abudayyeh, O.O., Barcena, C., Hsu, P.D., Habib, N., Gootenberg, J.S., Nishimasu, H., et al. (2015). Genome-scale transcriptional activation by an engineered CRISPRCas9 complex. Nature 517, 583-588. https://doi.org/10.1038/nature14136.

Krenciute, G., Liu, S., Yucer, N., Shi, Y., Ortiz, P., Liu, Q., Kim, B.-J., Odejimi, A.O., Leng, M., Qin, J., et al. (2013). Nuclear BAG6-UBL4A-GET4 complex mediates DNA damage signaling and cell death. J. Biol. Chem. 288, 2054720557. https://doi.org/10.1074/jbc.M112.443416.

Le Fourn, V., Girod, P.-A., Buceta, M., Regamey, A., and Mermod, N. (2014). $\mathrm{CHO}$ cell engineering to prevent polypeptide aggregation and improve therapeutic protein secretion. Metab. Eng. 21, 91-102. https://doi.org/10.1016/j. ymben.2012.12.003.

Lee, J.S., Kallehauge, T.B., Pedersen, L.E., and Kildegaard, H.F. (2015). Sitespecific integration in $\mathrm{CHO}$ cells mediated by CRISPR/Cas9 and homologydirected DNA repair pathway. Sci. Rep. 5, 8572. https://doi.org/10.1038/ srep08572.

Li, W., Xu, H., Xiao, T., Cong, L., Love, M.I., Zhang, F., Irizarry, R.A., Liu, J.S., Brown, M., and Liu, X.S. (2014). MAGeCK enables robust identification of essential genes from genome-scale CRISPR/Cas9 knockout screens. Genome Biol. 15, 554. https://doi.org/10.1186/s13059-014-0554-4.

Liao, Y., Wang, J., Jaihnig, E.J., Shi, Z., and Zhang, B. (2019). WebGestalt 2019: gene set analysis toolkit with revamped Uls and APIs. Nucleic Acids Res. 47, W199-W205. https://doi.org/10.1093/nar/gkz401.

Liu, Y., Cao, Z., Wang, Y., Guo, Y., Xu, P., Yuan, P., Liu, Z., He, Y., and Wei, W. (2018). Genome-wide screening for functional long noncoding RNAs in human cells by Cas 9 targeting of splice sites. Nat. Biotechnol. 36, 1203-1210. https:// doi.org/10.1038/nbt.4283.

Luo, H., Lin, Y., Gao, F., Zhang, C.-T., and Zhang, R. (2014). DEG 10, an update of the database of essential genes that includes both protein-coding genes and noncoding genomic elements: Table 1. Nucleic Acids Res. 42, D574D580. https://doi.org/10.1093/nar/gkt1131.

Mi, H., Muruganujan, A., Ebert, D., Huang, X., and Thomas, P.D. (2019a). PANTHER version 14: more genomes, a new PANTHER GO-slim and improvements in enrichment analysis tools. Nucleic Acids Res. 47, D419-D426. https://doi.org/10.1093/nar/gky1038.

Mi, H., Muruganujan, A., Huang, X., Ebert, D., Mills, C., Guo, X., and Thomas, P.D. (2019b). Protocol Update for large-scale genome and gene function analysis with the PANTHER classification system (v.14.0). Nat. Protoc. 14, 703-721. https://doi.org/10.1038/s41596-019-0128-8.

Mohan, C., and Lee, G.M. (2010). Effect of inducible co-overexpression of protein disulfide isomerase and endoplasmic reticulum oxidoreductase on the specific antibody productivity of recombinant Chinese hamster ovary cells. Biotechnol. Bioeng. 107, 337-346. https://doi.org/10.1002/bit.22781.
Petersen, S.D., Zhang, J., Lee, J.S., Jakočiūnas, T., Gray, L.M., Kildegaard, H.F., Keasling, J.D., and Jensen, M.K. (2018). 'Modular 5'-UTR hexamers for context-independent tuning of protein expression in eukaryotes'. Nucleic Acids Res. https://doi.org/10.1093/nar/gky734.

Prashad, K., and Mehra, S. (2015). Dynamics of unfolded protein response in recombinant $\mathrm{CHO}$ cells. Cytotechnology 67, 237-254. https://doi.org/10. 1007/s10616-013-9678-8.

Pristovšek, N., Nallapareddy, S., Gray, L.M., Hefzi, H., Lewis, N.E., Rugbjerg, P., Hansen, H.G., Lee, G.M., Andersen, M.R., and Kildegaard, H.F. (2019). Systematic evaluation of site-specific recombinant gene expression for programmable mammalian cell engineering. ACS Synth. Biol. 8, 758-774. https://doi. org/10.1021/acssynbio.8b00453.

Rajagopal, N., Srinivasan, S., Kooshesh, K., Guo, Y., Edwards, M.D., Banerjee, B., Syed, T., Emons, B.J.M., Gifford, D.K., and Sherwood, R.I. (2016). Highthroughput mapping of regulatory DNA. Nat. Biotechnol. 34, 167-174. https://doi.org/10.1038/nbt.3468.

Reiling, J.H., Clish, C.B., Carette, J.E., Varadarajan, M., Brummelkamp, T.R., and Sabatini, D.M. (2011). A haploid genetic screen identifies the major facilitator domain containing 2A (MFSD2A) transporter as a key mediator in the response to tunicamycin. Proc. Natl. Acad. Sci. U S A 108, 11756-11765. https://doi.org/10.1073/pnas.1018098108.

Rodriguez-Cuenca, S., Barbarroja, N., and Vidal-Puig, A. (2015). Dihydroceramide desaturase 1, the gatekeeper of ceramide induced lipotoxicity. Biochim. Biophys. Acta 1851, 40-50. https://doi.org/10.1016/j.bbalip.2014.09.021.

Rothe, M., Modlich, U., and Schambach, A. (2014). Biosafety challenges for use of lentiviral vectors in gene therapy. Curr. Gene Ther. 13, 453-468. https://doi.org/10.2174/15665232113136660006.

Rupp, O., MacDonald, M.L., Li, S., Dhiman, H., Polson, S., Griep, S., Heffner, K., Hernandez, I., Brinkrolf, K., Jadhav, V., et al. (2018). A reference genome of the Chinese hamster based on a hybrid assembly strategy. Biotechnol. Bioeng. 115, 2087-2100. https://doi.org/10.1002/bit.26722.

Sasaki, T., Gan, E.C., Wakeham, A., Kornbluth, S., Mak, T.W., and Okada, H. (2007). HLA-B-associated transcript 3 (Bat3)/Scythe is essential for p300mediated acetylation of p53'. Genes Dev. 21, 848-861. https://doi.org/10. 1101/gad.1534107.

Schambach, A., Zychlinski, D., Ehrnstroem, B., and Baum, C. (2013). Biosafety features of lentiviral vectors. Hum. Gene Ther. 24, 132-142. https://doi.org/10. 1089/hum.2012.229.

Sergeeva, D., Lee, G.M., Nielsen, L.K., and Gray, L.M. (2020). Multicopy targeted integration for accelerated development of high-producing Chinese hamster ovary cells. ACS Synth. Biol. 9, 2546-2561. https://doi.org/10.1021/ acssynbio.0c00322.

Siddique, M.M., Li, Y., Wang, L., Ching, J., Mal, M., Ilkayeva, O., Wu, Y.J., Bay, B.H., and Summers, S.A. (2013). Ablation of dihydroceramide desaturase 1, a therapeutic target for the treatment of metabolic diseases, simultaneously stimulates anabolic catabolic signaling. Mol. Cell Biol. 33, 2353-2369. https://doi.org/10.1128/MCB.00226-13.

Spahn, P.N., Bath, T., Weiss, R.J., Kim, J., Esko, J.D., Lewis, N.E., and Harmendy, O. (2017). PinAPL-Py: a comprehensive web-application for the analysis of CRISPR/Cas9 screens. Sci. Rep. 7, 15854. https://doi.org/10.1038/ s41598-017-16193-9.

Szklarczyk, D., Gable, A.L., Lyon, D., Junge, A., Wyder, S., Huerta-Cepas, J., Simonovic, M., Doncheva, N.T., Morris, J.H., Bork, P., et al. (2019). 'STRING v11: protein-protein association networks with increased coverage, supporting functional discovery in genome-wide experimental datasets'. Nucleic Acids Res. 47, D607-D613. https://doi.org/10.1093/nar/gky1131.

Tigges, M., and Fussenegger, M. (2006). Xbp1-based engineering of secretory capacity enhances the productivity of Chinese hamster ovary cells. Metab. Eng. 8, 264-272. https://doi.org/10.1016/j.ymben.2006.01.006.

Walsh, G. (2018). Biopharmaceutical benchmarks 2018. Nat. Biotechnol. 36, 1136-1145. https://doi.org/10.1038/nbt.4305.

Wang, J., Vasaikar, S., Shi, Z., Greer, M., and Zhang, B. (2017a). WebGestalt 2017: a more comprehensive, powerful, flexible and interactive gene set 
enrichment analysis toolkit. Nucleic Acids Res. 45, W130-W137. https://doi org/10.1093/nar/gkx356.

Wang, T., Yu, H., Hughes, N.W., Liu, B., Kendirli, A., Klein, K., Chen, W.W., Lander, E.S., and Sabatini, D.M. (2017b). Gene essentiality profiling reveals gene networks and synthetic lethal interactions with oncogenic ras. Cell 168, 890-903.e15. https://doi.org/10.1016/j.cell.2017.01.013.

Wang, T., Birsoy, K., Hughes, N.W., Krupczak, K.M., Post, Y., Wei, J.J. Lander, E.S., and Sabatini, D.M. (2015). Identification and characterization of essential genes in the human genome. Science 350, 1096-1101. https://doi. org/10.1126/science.aac7041.

Xiong, K., Marquart, K.F., Cour Karottki, K.J., Li, S., Shamie, I., Lee, J.S., Gerling, S., Yeo, N.C., Chavez, A., Lee, G.M., et al. (2019). Reduced apoptosis in Chinese hamster ovary cells via optimized CRISPR interference. Biotechnol. Bioeng. 116, 1813-1819. https://doi.org/10.1002/bit.26969.
Zha, W., Cao, L., Shen, Y., and Huang, M. (2013). Roles of Mir-144-ZFX Pathway in Growth Regulation of Non-Small-Cell Lung Cancer. PLoS One 8 e74175. https://doi.org/10.1371/journal.pone.0074175.

Zhou, Y., Su, Z., Huang, Y., Sun, T., Chen, S., Wu, T., Chen, G., Xie, X., Li, B., and $\mathrm{Du}, \mathrm{Z}$. (2011). The $\mathrm{Zfx}$ gene is expressed in human gliomas and is important in the proliferation and apoptosis of the human malignant glioma cell line U251. J. Exp. Clin. Cancer Res. 30, 114. https://doi.org/10.1186/1756-996630-114.

Zhu, Z., Li, K., Xu, D., Liu, Y., Tang, H., Xie, Q., Xie, L., Liu, J., Wang, H., Gong Y., et al. (2013). ZFX regulates glioma cell proliferation and survival in vitro and in vivo. J. Neuro Oncol. 112, 17-25. https://doi.org/10.1007/s11060-0121032-z.

Zhu, Q., Yang, J., Zhu, R., Jiang, X., Li, W., He, S., and Jin, J. (2016). Dihydroceramide-desaturase-1-mediated caspase 9 activation through ceramide plays a pivotal role in palmitic acid-induced HepG2 cell apoptosis. Apoptosis 21, 1033-1044. https://doi.org/10.1007/s10495-016-1267-9. 


\section{STAR $\star$ METHODS}

\section{KEY RESOURCES TABLE}

\begin{tabular}{|c|c|c|}
\hline REAGENT or RESOURCE & SOURCE & IDENTIFIER \\
\hline \multicolumn{3}{|l|}{ Deposited data } \\
\hline Raw and analyzed data & This paper & https://doi.org/10.5281/zenodo.4494299 \\
\hline $\begin{array}{l}\text { Raw NGS in NCBI SRA (also available in } \\
\text { the zenodo repo) }\end{array}$ & This paper & NCBI bioproject accession PRJNA699155 \\
\hline \multicolumn{3}{|l|}{ Experimental models: Cell lines } \\
\hline $\mathrm{CHO}-\mathrm{S}$ & Thermo Fisher Scientific & Cat R80007 \\
\hline \multicolumn{3}{|l|}{ Oligonucleotides } \\
\hline All oligos can be found in the zenodo repo & This paper & https://doi.org/10.5281/zenodo.4494299 \\
\hline \multicolumn{3}{|l|}{ Recombinant DNA } \\
\hline All plasmids can be found in the zenodo repo & This paper & https://doi.org/10.5281/zenodo.4494299 \\
\hline \multicolumn{3}{|l|}{ Software and algorithms } \\
\hline Pinapl-py & Spahn et al., 2017 & http://pinapl-py.ucsd.edu \\
\hline WEB-based GEne SeT AnaLysis Toolkit & $\begin{array}{l}\text { Wang et al., } 2017 a ; \\
\text { Liao et al., } 2019\end{array}$ & http://www.webgestalt.org/ \\
\hline STRING-DB & Szklarczyk et al., 2019 & https://string-db.org \\
\hline Panther-db & Mi et al., 2019a, 2019b & http://www.pantherdb.org/ \\
\hline
\end{tabular}

\section{RESOURCE AVAILABILITY}

\section{Lead contact}

Further information and requests for resources and reagents should be directed to and will be fulfilled by the Lead Contact, Lasse Ebdrup Pedersen (laeb@dtu.dk).

\section{Materials availability}

All unique/stable reagents generated in this study are available from the Lead Contact with a completed Materials Transfer Agreement.

\section{Data and code availability}

Sequencing data and analysis scripts are available at Zenodo https://doi.org/10.5281/zenodo.4494299.

Sequencing data is also deposited in the SRA at NCBI, bioproject accession PRJNA699155.

Remaining data is available in the supplemental information.

\section{EXPERIMENTAL MODEL AND SUBJECT DETAILS}

\section{Cell culture and transfection}

CHO-S cells (Thermo Fisher Scientific) were cultured in CD CHO medium supplemented with 8 mM L-Glutamine (Thermo Fisher Scientific) and $2 \mu \mathrm{L} / \mathrm{mL}$ Anti-Clumping reagent (AC, Life Technologies). Cells were maintained in Erlenmeyer shake flasks (Corning Inc., Acton, MA), incubated in a humidified incubator at $37^{\circ} \mathrm{C}, 5 \% \mathrm{CO} 2$ at $120 \mathrm{rpm}$ and passaged every 2-3 days. Viable cell density (VCD) and viability were monitored using the NucleoCounter NC-200 Cell Counter (ChemoMetec, Denmark). One day before transfection, cells were seeded into the culture medium without AC. On the day of transfection, cells were transfected using $3.75 \mu \mathrm{g}$ of DNA packaged with $3.75 \mu \mathrm{L}$ of FreeStyle Max transfection reagent (Thermo Fisher Scientific) per well of a 6 -well plate (BD Biosciences) in $3 \mathrm{~mL}$ culture medium without $\mathrm{AC}$ and with $1 \mathrm{E} 6 \mathrm{cells} / \mathrm{mL}$. Specifically, for VF gRNA library transfection, $150 \mu \mathrm{g}$ Bxb1 recombinase plasmid and $450 \mu \mathrm{g}$ gRNA library donor were transfected into $480 \mathrm{~mL}$ CHO-attp-mCherry cells at the density of $1 \mathrm{E} 6 \mathrm{cells} / \mathrm{mL}$. The cells were divided into several 6-well plates and one day after transfection, the cells were combined into two $500 \mathrm{~mL}$ flasks. Transfection efficiencies were monitored using the transfection reagent included pmax-GFP plasmid and was generally observed to be between $60-90 \%$. 


\section{METHOD DETAILS}

Expressed genes filtering and VF gRNA library design

RNA-seq data from $1558 \mathrm{CHO}$ cell samples were analyzed to identify all expressed $\mathrm{CHO}$ genes from a recent genome assembly and annotation (Rupp et al., 2018). 18238 genes were expressed at TPM $>1$ in at least $5 \%$ of the samples; 27 genes were expressed between $0.5-1$ TPM in at least $40 \%$ samples; 88 genes were expressed at $<0.5$ TPM in $85 \%$ samples. gRNAs were designed by DESKTOP Genetics based on the CH genome (Rupp et al., 2018) and proprietary Pacific Biosciences genome sequence data from CHO-S. This resulted in a library containing 75488 unique gRNAs of which 72149 target 15028 genes, 2218 target intergenic regions, 1051 are non-targeting gRNAs and finally $70 \mathrm{gRNA}$ are targeting multiple genes (Table S1). gRNAs oligos were synthesized by Twist Biosciences (San Francisco, CA). The annealing oligos were cloned into the attB-Esp3I vector (See plasmid map and sequence) to make an attB-gRNA vector as donor DNA for RMCE following a protocol described previously (Joung et al., 2017).

\section{Lentiviral library generation}

The lentiviral cell library was made in a previous study (Karottki et al., 2021) where wild type CHO-S cells (Thermo Fisher Scientific) were transduced with the lentiviral library. Briefly, gRNAs involved in $\mathrm{CHO}$ cell metabolism were designed, synthesized, and packaged into lentivirus. The lentiviral library was used to transduce $\mathrm{CHO}-\mathrm{S}$ cells at a low virus to cell ratio $(\mathrm{MOI}=0.25)$. The transduced cells were subject to puromycin selection to remove cells that did not receive a gRNA cassette. In order to facilitate comparison with our viral free library, we re-annotated these gRNAs. The lentiviral library consists of 16985 unique gRNAs of which 16744 target 2909 genes, 145 target intergenic regions, 89 are non-targeting gRNA and 7 gRNAs are targeting multiple genes (Table S2).

CHO-attp-mCherry cell line generation with RMCE landing pad and the RMCE of gRNA expression cassette The CHO-attp-mCherry cell line was made CRISPR-mediated homology directed targeted integration of a RMCE landing pad into T2 genomic site in CHO-S cells (Thermo Fisher Scientific) as previously described (Lee et al., 2015; Petersen et al., 2018; Pristovšek et al., 2019). This cell line contains an mCherry coding sequence flanked by an attp sequence at the 5 ' end and a mutant attp sequence at the 3' end (pEF1a-attp-mCherry-HygroR-attp, landing pad vector is shown in plasmid map and sequence), and the 5' and 3' homology arms target at a transcriptionally active site within a non-coding region (T2 site). Vectors for RMCE were constructed by assembly of PCR fragments containing promoter-less puroR gene and gRNA cassette that was flanked by attB and mutant attB sequences. The cell with landing pad was transfected with RMCE gRNA plasmid (library) and Bxb1-recombinase vector in 3:1 ratio to exchange mCherry coding sequence with gRNA cassette. For Bxb1 recombinase expression, PSF-CMV-Bxb1 recombinase vector (see plasmid and sequence) was used. To maintain enough gRNA coverage (500 copies per gRNA), $480 \mathrm{~mL}$ (1E6 viable cells $/ \mathrm{mL}$ ) of cells were transfected (while RMCE rate is measured as $4 \%$ in Figure S1). Three days after transfection, the transfected cell pool was selected with $10 \mu \mathrm{g} / \mathrm{mL}$ puromycin. After 14-20 days, the cell pool was fully enriched to mCherry negative populations. The transfected cell pool was passaged every two days. After 9 and 25 days of gRNA RMCE, cells were analyzed by flow cytometry (BD FACSJazz cell sorter, BD Biosciences) to measure the mCherry density in the cell pool. The percentages of mCherry-negative cells on day 9 and day 25 were calculated to estimate the RMCE efficiency and confirm the cell-based gRNA library was completely established.

\section{Copy number analysis using qPCR}

Relative copy numbers of the integrated gRNA were determined by qPCR. Genomic DNA was extracted using the GeneJET Genomic DNA Purification Kit (Thermo Fisher Scientific) according to manufacturer instructions. qPCR was run on a QuantStudio 5 Real-Time PCR System (Agilent Technologies). Amplification was performed under the following conditions: $50^{\circ} \mathrm{C}$ for 2 min, $95^{\circ} \mathrm{C}$ for $10 \mathrm{~min}$; 40x: $95^{\circ} \mathrm{C}$ for $15 \mathrm{~s}, 60^{\circ} \mathrm{C}$ for $1 \mathrm{~min}$. Copy numbers of gRNA were determined with C1GALT1C1 as an internal control gene for normalization using SYBR Green qPCR kit (Thermo Fisher Scientific). Forward primer $5^{\prime}$-GCAGCCTTCTATCTAGGACAC-3' and reverse primer 5'-CCACCTTGTTCAGGACACTT-3' are designed for C1GALT1C1 detection. Forward primer 5'-GCTTTATATATCTTGTG GAAAGGACGAAACACC-3' and reverse primer $5^{\prime}$-CCGACTCGGTGCCACTITTCAA-3' are designed for gRNA detection. Primer pairs were validated by melting curve analysis and primer efficiency test. A delta-delta threshold cycle $(\Delta \Delta \mathrm{Ct})$ method was applied to calculate the copy number of gRNA integrated using previously single-copy calibrators. Each experiment was performed in technical triplicates using genomic DNA from $\mathrm{CHO}-\mathrm{S}$ cells as a negative control.

\section{Cas9-transfected cells enrichment}

The cell-based gRNA library transfected with GFP was used as control. One day after transfection of Cas9-BSD vector (See plasmid map and sequence), the cells were treated with $10 \mu \mathrm{g} / \mathrm{mL}$ of blasticidin for 1 day and with $5 \mu \mathrm{g} / \mathrm{mL}$ of blasticidin for an additional one day. On day 4 after transfection the cells were cultured in medium without blasticidin for recovery. After day 8 , the recovered cell pool was ready to be used for experiments. Cells in the control group were re-seeded to $1 \mathrm{E} 6 \mathrm{cells} / \mathrm{mL}$ whenever the viable cell density reached $5 \mathrm{E} 6$ cells $/ \mathrm{mL}$.

\section{Preparation for NGS}

$50 \mu \mathrm{L}$ PCR reactions with 2.5-3 $\mu$ g input gDNA per reaction were run using Phusion ${ }^{\circledR}$ Hot Start II High-Fidelity DNA Polymerase (Thermo Fisher Scientific) $\left(95^{\circ} \mathrm{C}\right.$ for $3 \mathrm{~min} ; 30$ times: $98^{\circ} \mathrm{C}$ for $45 \mathrm{~s}, 60^{\circ} \mathrm{C}$ for $30 \mathrm{~s}, 72^{\circ} \mathrm{C}$ for $1 \mathrm{~min}$; $72^{\circ} \mathrm{C}$ for 7 min) using primers identical 


\section{Cell Reports Methods}

OPEN ACCESS

in the method of copy number analysis. PCR products were purified by Macherey-Nagel NucleoSpin Gel \& PCR purification kit. The amplicons were added with Illumina NGS index with adapters using DNA ligation. The resulting library was quantified with Qubit using the dsDNA HS Assay Kit (Thermo Fisher Scientific) and the fragment size was determined using a 2100 Bioanalyzer Instrument (Agilent) before running the samples on a NextSeq 500 sequencer (Illumina).

Clone variance analysis

Single cells were sorted from the LV-lib pool (before Cas9 transfection) and VF library pool (CHO-S cells infected with lentiviral gRNA library but without Cas9) and deposited into a 96-well plate by BD FACSJazz cell sorter (BD Biosciences). 14 days after single cell sorting, the confluence of the emerging colonies in each well was measured by Celigo Cell Imaging Cytometer (Nexcelom Bioscience). Colonies with confluence lower than $10 \%$ were filtered out. The clones of 4 plates from VF library and 4 plates from LV-lib was calculated.

\section{Gene disruption validation}

One new gRNA for each gene was designed to target Bag6 and Zfx gene, respectively. The target sequences of gRNAs targeting Bag6, Zfx are GGCATTCCGGTCATGAACAGAGG (Bag6-gRNA) and TTCTTCTGAAACAACATCGGCGG (Zfx-gRNA), respectively. Detailed materials and methods for gRNA design and the gRNA vector construction are elaborated in our previous study (Xiong et al., 2019). The CHO-Cas9 cells with stable expression of Cas9 was transfected with gRNAs. 7 days after transfection, the recovered cells were cultured in the medium described as above with $20 \mathrm{ng} / \mathrm{mL}$ tunicamycin (TM) for 4 days as indicated in legends. The $\mathrm{CHO}-\mathrm{Cas} 9$ cells transfected with GFP or NT-gRNA were treated with $20 \mathrm{ng} / \mathrm{mL} \mathrm{TM}$ as control. The medium was never changed during these 4 days of TM treatment. The cell viability and the VCD were measured using the NucleoCounter NC-200 Cell Counter (ChemoMetec, Denmark) during this TM treatment process. A non-targeting gRNA was used as control as described previously (Karottki et al., 2020).

Generation of CHO-Cas9 cell line and CHO-attP-mCherry/Cas9 cell line

To establish a cell platform for stably expressing Cas9, a CHO-Cas9 cell line was generated from a parent cell line containing a RMCE landing pad. The parent cell line was made by CRISPR-mediated homology directed targeted integration of $\mathrm{CHO}-\mathrm{S}$ cells (Life Technologies) as previously described (Lee et al., 2015; Petersen et al., 2018; Pristovšek et al., 2019). The parent cell line contains a mCherry coding sequence flanked by a loxP sequence at the 5' end and a lox2272 sequence at the 3' end (pEF1-loxP-mCherry- lox2272-BGHpA), and the 5' and 3' homology arms target a transcriptionally active site within a noncoding region (See plasmid map and sequence). Promoter-less RMCE vectors were constructed by assembly of PCR fragments containing the Cas9 region flanked by loxP and lox2272 sequences. The parent cell line was transfected with RMCE Cas9 donor plasmids (See plasmid map and sequence) and Cre-recombinase vector in 3:1 ratio to exchange the mCherry coding sequence with Cas9. For Cre recombinase expression, PSF-CMV-CRE recombinase vector (OGS591, Sigma-Aldrich) was used. Transfected cell pool was passaged twice after transfection. After 7 days, mCherry-negative single cell sorting was performed by FACS using a BD FACSJazz cell sorter (BD Biosciences). After 14 days, the monoclonal CHO-Cas 9 cell line was established after verification of proper integration and confirmation of single copy of Cas9. The methods and the materials for generating this $\mathrm{CHO}-\mathrm{Cas} 9$ cell line were described before in our study (Hart et al., 2015). By using CHO-Cas9 cell line as the parent cell line, attP RMCE landing pad was inserted into the T2 site as the protocol described above to generate $\mathrm{CHO}-\mathrm{attP}-\mathrm{mCherry} / \mathrm{Cas} 9$ cell line.

gRNA design for Mgat1 KO and Mgat1 editing analysis

A gRNA targeting Mgat1 (gRNA-Mgat1) was designed to target GTGGAGTTGGAGCGGCAGCGGGG. gRNA-Mgat1 was cloned into the attB vector to achieve RMCE of gRNA-Mgat1 showed in Figures 1H and S2. gRNA-Mgat1 was cloned into identical gRNA vector as described in our previous study for Mgat1 KO by transient expression in Figure $1 \mathrm{H}$. A pair of primers with the sequence 5'-TTCTGGACACGCCCAGC-3' (forward) and 5'-GCCACGGTGGGCACTTT-3' was applied to detect Mgat1 editing by Sanger sequencing (Figure S2) or by NGS analysis (Figure1H). A non-targeting gRNA was used as control for confirming the Mgat1 KO (Figure S2).

Establishment of Zfx KO cell lines

CHO-S WT cells or dual-RMCE ETN2_T9 CHO cells were transfected with Zfx-gRNA described above. The pools were single cell sorted by FACS into 96-well cell culture plates. After 14 days, the single cell derived populations were tested for double KO of Zfx using sanger sequencing of amplicons generated with the following primers: forward: $5^{\prime}-$ TACATGTGGCTGACGTTGGT-3' and reverse: 5'- GGAAATCATAAGGTAGTCCTCACA-3'. CHO-S WT cells and dual-RMCE ETN2_T9 cells transfected with NT-gRNA and otherwise processed identically as above were used as control (Figures $7 \mathrm{G}$ and $7 \mathrm{H}$ ).

Plasmid maps and sequences

Sequences and maps of all plasmids can be found in the zenodo data repository https://doi.org/10.5281/zenodo.4494299 


\section{QUANTIFICATION AND STATISTICAL ANALYSIS}

\section{NGS data analysis}

The gRNA coverage is calculated by the percentage of gRNA with at least 1 read in the library and skew ratio analysis was calculated by the ratio of reads at cumulative percentage $90 \%$ to $10 \%$, following suggestions in former CRISPR GeCKO library screens protocol from Feng Zhang's lab (Konermann et al., 2015; Joung et al., 2017). For gRNA fold change analysis, raw FASTQ files were analyzed using default parameters in PinAPL-PY (Spahn et al., 2017) (http://pinapl-py.ucsd.edu/) along with a file containing the sequences for all gRNAs contained in the library. Top candidates for enriched and for depleted gRNAs were ranked by the adjusted robust rank aggregation (aRRA) method (Li et al., 2014) and filtered for significance, compared between the replicates and used for verification of the screen. A gene was labelled "significant" if it was both statistically significant on the gene levels as determined by pinapl-py and statistically significant on the gRNA level for at least $3 \mathrm{gRNAs}$ and at least half the gRNAs targeting the gene. Biological process depletion was performed in the PANTHER classification system (Mi et al., 2019a, 2019b) (http://www.pantherdb.org). The list of gRNA fold change was tested against the PANTHER GO-Slim Biological Process annotation set. Network topology-based analysis based on protein-protein-interaction (PPI) of candidate genes was performed in WEB-based GEne SeT AnaLysis Toolkit (Wang et al., 2017a; Liao et al., 2019). Network Retrieval \& Prioritization method was applied in this NTA analysis (http://www.webgestalt.org). The PPI analysis was confirmed in STRING database (string-db.org) (Szklarczyk et al., 2019). Essential genes in human and mouse were obtained from the DEG database (Luo et al., 2014) for essential genes (http://tubic.tju.edu.cn/deg). Essential gene lists of K562 cell and Hela cell were obtained from previous CRISPR screen studies (Hart et al., 2015; Wang et al., 2015) collected in the essential gene database (http://www.essentialgene.org/).

Raw data, PinAPL-PY output, and data analysis scripts can be downloaded from https://doi.org/10.5281/zenodo.4494299 Details of statistical tests can be found in relevant figure legends. 